Article

\title{
Phosphonium-Based Porous Ionic Polymer with Hydroxyl Groups: A Bifunctional and Robust Catalyst for Cycloaddition of $\mathrm{CO}_{2}$ into Cyclic Carbonates
}

\author{
Yizhu Lei ${ }^{1, *}$, Yali Wan ${ }^{2, *}$, Wei Zhong ${ }^{1}$, Dingfu Liu ${ }^{2}$ and Zhou Yang ${ }^{1}$ \\ 1 School of Chemistry and Materials Engineering, Liupanshui Normal University, Liupanshui 553004, China; \\ zw18385142340@163.com (W.Z.); YangZhouand@163.com (Z.Y.) \\ 2 School of Chemistry and Chemical Engineering, Guizhou University, Guiyang 550025, China; \\ liudfgu@163.com \\ * $\quad$ Correspondence: yzleiabc@126.com (Y.L.); yliwan06@163.com (Y.W.); Tel.: +86-085-8860-0172 (Y.L.)
}

Received: 9 February 2020; Accepted: 3 March 2020; Published: 5 March 2020

\begin{abstract}
The integration of synergic hydrogen bond donors and nucleophilic anions that facilitates the ring-opening of epoxide is an effective way to develop an active catalyst for the cycloaddition of $\mathrm{CO}_{2}$ with epoxides. In this work, a new heterogeneous catalyst for the cycloaddition of epoxides and $\mathrm{CO}_{2}$ into cyclic carbonates based on dual hydroxyls-functionalized polymeric phosphonium bromide (PQPBr-2OH) was presented. Physicochemical characterizations suggested that $\mathrm{PQPBr}-2 \mathrm{OH}$ possessed large surface area, hierarchical pore structure, functional hydroxyl groups, and high density of active sites. Consequently, it behaved as an efficient, recyclable, and metal-free catalyst for the additive and solvent free cycloaddition of epoxides with $\mathrm{CO}_{2}$. Comparing the activity of $\mathrm{PQPBr}-2 \mathrm{OH}$ with that of the reference catalysts based on mono and non-hydroxyl functionalized polymeric phosphonium bromides suggested that hydroxyl functionalities in $\mathrm{PQPBr}-2 \mathrm{OH}$ showed a critical promotion effect on its catalytic activity for $\mathrm{CO}_{2}$ conversion. Moreover, $\mathrm{PQPBr}-2 \mathrm{OH}$ proved to be quite robust and recyclable. It could be reused at least ten times with only a slight decrease of its initial activity.
\end{abstract}

Keywords: porous ionic polymer; heterogeneous catalysis; $\mathrm{CO}_{2}$ fixation; hydrogen bond donors; phosphonium salt

\section{Introduction}

With the increasing concerns about environmental crisis, the development of efficient routes for the capture, storage, and utilization of carbon dioxide $\left(\mathrm{CO}_{2}\right)$ that mitigates global warming has attracted tremendous attentions [1,2]. In this context, numerous strategies for mitigating $\mathrm{CO}_{2}$ emissions have been proposed, among which the conversion of $\mathrm{CO}_{2}$ into useful chemicals appears promising [3,4]. Of various $\mathrm{CO}_{2}$ chemical conversions, the catalytic cycloaddition of epoxides and $\mathrm{CO}_{2}$ into cyclic carbonates is regarded as one of the most efficient reactions due to its easily available substrates, high selectivity, and 100\% atom efficiency [5,6]. At present, many homogeneous catalytic systems have been developed to promote this conversion. Among them, the homogeneous catalysts based on ionic liquids, such as quaternary ammonium salts [7,8], imidazolium salts [9-11], and quaternary phosphonium salts [12-14], were demonstrated to be the most efficient. Generally, the homogeneous salts could exhibit high catalytic activity under mild reaction conditions; however, the obvious drawbacks of product separation and catalyst recycling still hamper their large-scale applications. Hence, it is desirable to develop highly efficient heterogeneous catalysts for the conversion of epoxides into cyclic carbonates. 
Porous organic polymers (POPs), featuring adjustable chemical functionalities and large surface areas, have recently aroused great interest as a class of versatile materials for developing heterogeneous catalysts $[15,16]$. Nowadays, a number of POPs have been developed for $\mathrm{CO}_{2}$ capture and conversion $[17,18]$. However, the majority of POPs contain monofunctional active sites. Thus, cocatalysts, such as ammonium salts, metal compounds, and/or hydrogen bond donors, are often added for achieving acceptable catalytic activities $[18,19]$. To heterogenize both catalyst and co-catalyst, bifunctional POPs that consisted of both nucleophiles (halogen anions) and electrophiles (metal salts or hydrogen bond donors) have been recently developed [20-25]. Previous reports suggested that POPs that have such dual active sites could cooperatively activate the epoxide, thus improving catalytic performances significantly [20-25]. For numerous types of POPs, porous ionic polymers (PiPs) that incorporated ionic moieties into POPs are particularly appropriate for cocatalyst-free $\mathrm{CO}_{2}$ fixation [26-29]. On this account, a number of PiPs with metal and halogen dual active sites have recently emerged as heterogeneous and efficient catalysts for $\mathrm{CO}_{2}$ conversion [29-32]. In contrast, from an environmental viewpoint, the cycloaddition of $\mathrm{CO}_{2}$ over a metal-free catalyst is more in line with the requirements of green chemistry because of the low toxicity and easy accessibility of these catalysts $[20,26,27,33-38]$. Therefore, the development of metal-free porous ionic polymer catalysts for the efficient cycloaddition of epoxides and $\mathrm{CO}_{2}$ into cyclic carbonates is still highly required.

With this target, a number of imidazolium-based PIPs that contain hydrogen bond donors (HBD) (such as hydroxyl, carboxylic acid, and amine groups) and halogen anions have recently been developed as heterogeneous catalysts for $\mathrm{CO}_{2}$ fixation $[20,26,27,33-38]$. Compared with imidazolium-based PIPs, quaternary phosphonium-based PIPs are not as extensively studied due to the less tailored structures and limited heterogenizing method [39,40]. In 2012, Zhang et al. reported that a porous organic polymer containing quaternary phosphonium and tertiary phosphorus (PP-Br) could act as an efficient and recyclable catalyst for the cycloaddition of $\mathrm{CO}_{2}$ [41]. In 2015, Wang et al. also reported that in the presence of $\mathrm{ZnBr}_{2}$, the phosphonium salt incorporated hypercrosslinked porous polymer displayed high catalytic activity [42]. Later on, Sun et al. synthesized quaternary phosphonium-based PIPs through the free-radical polymerization of vinyl-functionalized quaternary phosphonium salts [43]. The prepared polymeric quaternary phosphonium salts that featured a high density of halide ions exhibited high activities and reusability. Very recently, Xiao et al. reported that polymeric quaternary phosphonium salts containing bromide ion and phenolic hydroxyl group (PPS- $m \mathrm{OH}-\mathrm{Bn}$ ) exhibited an excellent synergistic effect for the cycloaddition of epoxides and $\mathrm{CO}_{2}$ [44]. The above results indicated that those functionalized phosphonium-based PIPs showed good catalytic performances and reusability for the cycloaddition of $\mathrm{CO}_{2}$, especially polymeric quaternary phosphonium salts with hydroxyl groups.

On the other hands, as a kind of easily available and robust HBD group, alkyl hydroxyls have also been widely incorporated in imidazolium-based PIPs [33-38] and quaternary phosphonium salts [14,40]; these previous works demonstrated that alkyl hydroxyls showed a nice promoting effect for imidazolium and quaternary phosphonium based catalysts. Inspired by these advances, we envisioned that the fabrication of polymeric quaternary phosphonium salts with alkyl hydroxyl groups might be an efficient way to construct bifunctional and heterogeneous catalysts for the cycloaddition of epoxides and $\mathrm{CO}_{2}$. Therefore, we herein designed and fabricated phosphonium-based porous ionic polymer with hydroxyl groups (PQPBr-2OH) as a bifunctional catalyst for $\mathrm{CO}_{2}$ fixation. As shown in Scheme 1, PQPBr-2OH was facilely prepared through the reaction of poly(tris(4-vinylphenyl)phosphine) $\left(\mathrm{POL}_{-} \mathrm{PPh}_{3}\right)$ and 1-bromo-2,3-epoxypropane, followed by ring opening in hot water. This dual hydroxyls-functionalized polymeric phosphonium bromide has a large surface area and a hierarchical pore structure. Consequently, $\mathrm{PQPBr}-2 \mathrm{OH}$ acted as an efficient, robust, and metal-free solid catalyst for the cycloaddition of epoxides with $\mathrm{CO}_{2}$. 


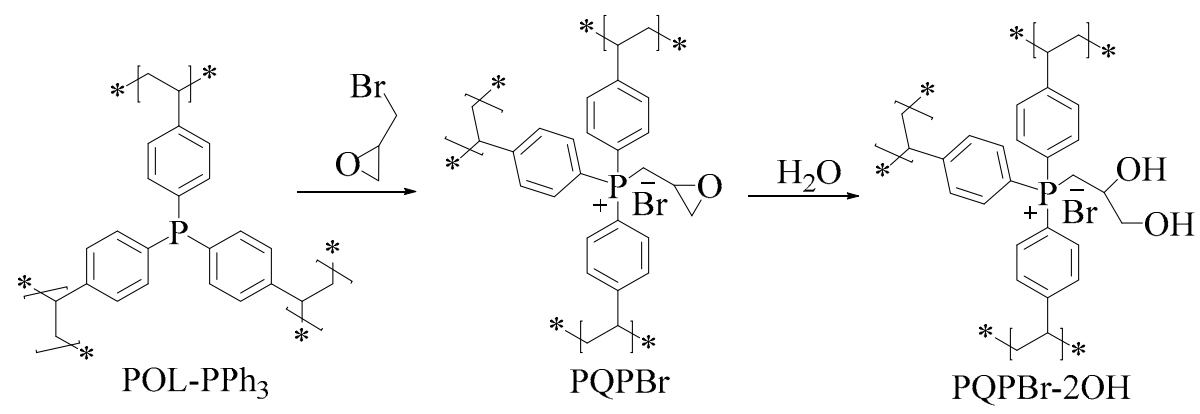

Scheme 1. Preparation of the PQPBr-2OH catalyst.

\section{Materials and Methods}

\subsection{Materials}

Epoxides, 1-bromo-2,3-epoxypropane, 3-bromo-1-propanol, tetrahydrofuran, and ethanol were purchased from Energy Chemical Company (Shanghai, China). $\mathrm{CO}_{2}$ (99.99\%) was supplied by a local manufacturer (Liupanshui, China). Tris(4-vinylphenyl)phosphane and poly(tris(4-vinylphenyl)phosphine) $\left(\mathrm{POL}-\mathrm{PPh}_{3}\right)$ were prepared as previously reported [45].

\subsection{Catalyst Preparation}

Epoxy-functionalized porous ionic polymer (PQPBr) was prepared via the reaction of poly(tris(4-vinylphenyl)phosphine) $\left(\mathrm{POL}-\mathrm{PPh}_{3}\right)$ and 1-bromo-2,3-epoxypropane. Generally, $1.0 \mathrm{~g}$ of $\mathrm{POL}_{-\mathrm{PPh}}$ was added in $30 \mathrm{~mL}$ anhydrous tetrahydrofuran (THF) in a $100 \mathrm{~mL}$ pressure-resistant glass tube. After magnetic stirring at room temperature for $2 \mathrm{~h}, 0.50 \mathrm{~g}$ of 1-bromo-2,3-epoxypropane was added to the mixture. Then, the mixture was stirred in an oil bath at $100{ }^{\circ} \mathrm{C}$ for $24 \mathrm{~h}$. After the reaction, the white solid was separated by centrifugation, washed with ethanol four times, and dried at $60{ }^{\circ} \mathrm{C}$ in a vacuum. The obtained solid was PQPBr. The elemental composition of PQPBr was: C, $68.52 \% ; \mathrm{H}, 5.57 \%$; O, 3.58\%; P, 6.38\%; Br, 14.21\%.

Mono hydroxyl-functionalized porous ionic polymer $(\mathrm{PQPBr}-\mathrm{OH})$ was prepared via the reaction

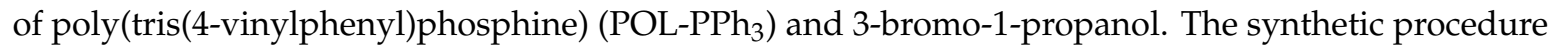
of PQPBr-OH was the same as that of PQPBr. The elemental composition of PQPBr-OH was: C, 67.85\%; $\mathrm{H}, 5.95 \%$; O, 3.65\%; P, 6.52\%; Br, 14.37\%.

Dual hydroxyls-functionalized porous ionic polymer $(\mathrm{PQPBr}-2 \mathrm{OH})$ was prepared by the ring opening reaction in hot water [46]. In a typical procedure, PQPBr $(1.0 \mathrm{~g})$ and water $(30 \mathrm{~mL})$ were added into a $100 \mathrm{~mL}$ pressure-resistant glass tube. The mixture was stirred at $80{ }^{\circ} \mathrm{C}$ for $24 \mathrm{~h}$, separated by filtration, and dried at $70^{\circ} \mathrm{C}$ in a vacuum oven (YihengDZF-6050, Shanghai Yiheng Technology Co., Ltd., Shanghai, China). The elemental composition of PQPBr-2OH was: C, 65.91\%; H, 5.73\%; O, 7.02\%; $\mathrm{P}, 6.29 \%$; $\mathrm{Br}, 14.15 \%$.

\subsection{Synthesis of Cyclic Carbonates}

In a representative experiment, epoxide and $\mathrm{PQPBr}-2 \mathrm{OH}(0.05-0.5 \mathrm{~mol} \%$, based on $\mathrm{Br}$ ion) was introduced into a $15 \mathrm{~mL}$ stainless-steel reactor. After purging with $\mathrm{CO}_{2}$ three times, the reactor was pressurized with $\mathrm{CO}_{2}$ to a low pressure $(0.5 \mathrm{MPa})$. Then, the reaction was proceeded at $60-140{ }^{\circ} \mathrm{C}$ for $4 \mathrm{~h}$. After the reaction, the reaction mixture was separated by centrifugation. The resulting solid was washed with ethanol, and dried under vacuum. The liquid mixture was qualitatively and quantitatively analyzed with gas chromatography (GC) and gas chromatography-mass spectrometry (GC-MS).

\subsection{Catalyst Recycling}

The recycling experiment of PQPBr-2OH was carried out in a ten-run cycling experiment. For facilitating the cycling of $\mathrm{PQPBr}-2 \mathrm{OH}$, the catalytic reaction was performed in scaled-up quantities 
in a $50 \mathrm{~mL}$ stainless-steel reactor. Typically, styrene oxide $(12 \mathrm{~g}, 100 \mathrm{mmol})$ and the catalyst $(0.11 \mathrm{~g}$, $0.2 \mathrm{~mol} \%$ ) were allowed to react at $120^{\circ} \mathrm{C}$ for $4 \mathrm{~h}$ with a consistent $\mathrm{CO}_{2}$ pressure of $0.5 \mathrm{MPa}$. After each catalytic run, the solid catalyst was recovered by centrifugation, washed with ethanol three times, and dried at $60{ }^{\circ} \mathrm{C}$ under vacuum. Then, the recovery PQPBr-2OH was reused in a new catalytic run.

\subsection{Characterizations}

Brunauer-Emmett-Teller (BET) surface areas of the samples were collected on Micrometrics ASAP 2020 (Micromeritics Instrument Co., Norcross, GA, USA) at $77 \mathrm{~K}$. Before the measurements, the samples were vacuum dried at $90{ }^{\circ} \mathrm{C}$ for $12 \mathrm{~h}$. Surface morphologies of the obtained samples were observed on scanning electron microscope (SEM, Hitachi S-4800, Hitachi Ltd., Tokyo, Japan) and transmission electron microscopy (TEM, FEI Tecnai G2 F30, Philips-FEI Co., Amsterdam, Netherlands). The contents of CHON elements in the samples were analyzed by an organic elemental analyzer (Elementar Vario MICRO, Elementar Analysensysteme, Hanau, Germany). The contents of P and Br elements were analyzed by inductive coupled plasma optima optical emission spectroscopy (ICP-OES, PerkinElmer, Optima 2000DV, Shelton, WA, USA) and ion chromatograph (Thermofisher ICS-2100, Thermo Fisher Scientific, Sunnyvale, CA, USA), respectively. Before measurement, the polymer was combusted in an oxygen bomb calorimeter and then dissolved in water for analyses. Solid-state ${ }^{13} \mathrm{C}$ and ${ }^{31} \mathrm{P}$ nuclear magnetic resonance (NMR) spectra were collected on a Bruker AVANCE III 600 Bruker spectrometer (Bruker Corp., Rheinstetten, Germany). X-ray photoelectron spectroscopy (XPS) was conducted on a VG multilab 2000 X-ray photoelectron spectrometer (Thermo Electron Corporation, Waltham, MA, USA). Thermal stability of the sample was tested by thermogravimetric analysis (TGA, NETZSCH STA 449 F5, Netzsch, Serb, Germany) over a temperature range from 30 to $600{ }^{\circ} \mathrm{C}$ at a heating rate of $10{ }^{\circ} \mathrm{C} / \mathrm{min}$ under Ar atmosphere. GC-MS analysis was performed in an Agilent 6890/5973 GC-MS apparatus (Agilent Technologies, Santa Clara, CA, USA). Gas chromatography analysis was carried out by Scientific ${ }^{\mathrm{TM}}$ TRACE ${ }^{\mathrm{TM}} 1310$ (Thermo Electron Corporation, Waltham, MA, USA) equipped with an FID detector and a TRACE TR-5MS capillary column.

\section{Results}

\subsection{Characterizations of the Polymeric Catalysts}

Dual hydroxyls-functionalized porous ionic polymer (PQPBr-2OH) was prepared by the reaction of poly(tris(4-vinylphenyl)phosphine) ( $\left.\mathrm{POP}-\mathrm{PPh}_{3}\right)$ and 1-bromo-2,3-epoxypropane, followed by the ring opening of the epoxy-tethered precursor $(\mathrm{PQPBr})$ in hot water. For comparison, mono hydroxyl-functionalized porous ionic polymer (PQPBr-OH) was also prepared. The molecular structure diagrams of the three catalysts, $\mathrm{PQPBr}-2 \mathrm{OH}, \mathrm{PQPBr}$, and PQPBr-OH, is illustrated in Scheme 2. The structure, morphologies, and properties of the obtained catalysts were carefully characterized by $\mathrm{N}_{2}$ adsorption-desorption, SEM, TEM, FT-IR, elemental analysis, Solid-state ${ }^{13} \mathrm{C}$ NMR and ${ }^{31}$ P NMR spectra, XPS, SEM-mapping, and TGA.
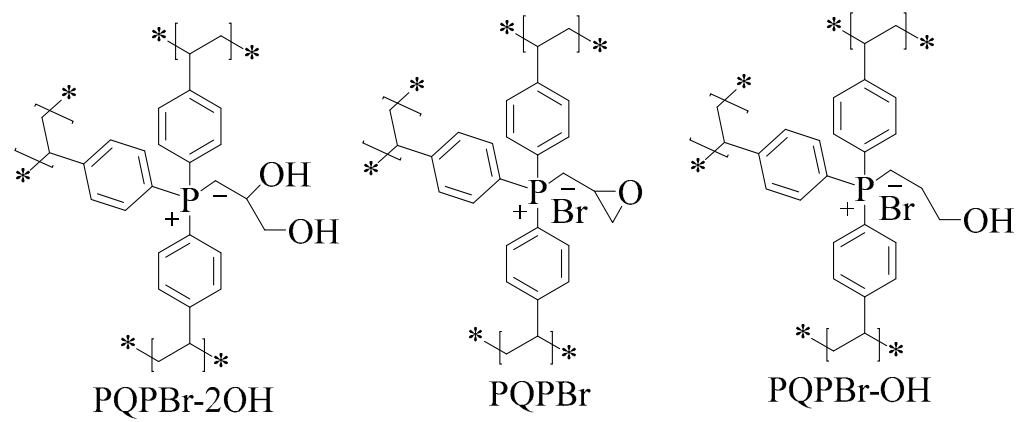

Scheme 2. Molecular structure diagrams of the prepared catalysts, ${ }^{*}$ means the long molecular chain. 
The porous properties of $\mathrm{PQPBr}, \mathrm{PQPBr}-\mathrm{OH}$, and $\mathrm{PQPBr}-2 \mathrm{OH}$ were analyzed by nitrogen sorption analysis. As shown in Table 1, $\mathrm{POP}-\mathrm{PPh}_{3}$ showed a high BET surface area of $1146 \mathrm{~m}^{2} \mathrm{~g}^{-1}$ and a pore volume of $2.41 \mathrm{~cm}^{3} \mathrm{~g}^{-1}$. After functionalization with epoxy, mono hydroxyl, and dual hydroxyls groups, the BET surface areas and pore volumes of the resulting $\mathrm{PQPBr}, \mathrm{PQPBr}-\mathrm{OH}$, and $\mathrm{PQPBr}-2 \mathrm{OH}$ obviously decreased. These decreases of physical properties could be assigned to the partial pore filling after functionalization with alkylation groups. In spite of the obvious decrease in BET surface areas with this post-modification method, the obtained PQPBr, $\mathrm{PQPBr}-\mathrm{OH}$, and $\mathrm{PQPBr}-2 \mathrm{OH}$ still possessed higher surface areas than that of polymeric quaternary phosphonium salts preparing by direct polymerization of vinyl-functionalized quaternary phosphonium salts in $\mathrm{N}, \mathrm{N}$-dimethylformamide (DMF) solvent [43,44]. These high BET surface areas might be attributed to the use of THF as a solvent for the polymerization and alkylation reactions; THF showed excellent swelling properties towards the phosphine-functionalized porous polymer that was prepared by the polymerization of vinyl-functionalized monomers $[47,48]$.

Table 1. Textural properties and $\mathrm{Br}^{-}$contents of the prepared catalysts.

\begin{tabular}{ccccc}
\hline Entry & Sample & $S_{\text {BET }}\left(\mathbf{m}^{\mathbf{2}} \mathbf{g}^{-\mathbf{1}}\right)^{a}$ & $V_{\mathbf{p}}\left(\mathbf{c m}^{\mathbf{3}} \mathbf{g}^{-\mathbf{1}}\right)^{b}$ & $\mathbf{D}_{\text {ave }}(\mathbf{n m})^{c}$ \\
\hline 1 & POP-PPh $_{3}$ & 1146 & 2.41 & 8.42 \\
2 & PQPBr & 879 & 2.20 & 6.25 \\
3 & PQPBr-OH & 848 & 1.57 & 3.39 \\
4 & PQPBr-2OH & 794 & 1.90 & 6.26
\end{tabular}

${ }^{a}$ BET surface area. ${ }^{b}$ Single point adsorption total pore volume of pores at $\mathrm{P} / \mathrm{Po}=0.95 .{ }^{c}$ Adsorption average pore diameter (4V/A by BET).

Nitrogen sorption isotherms of $\mathrm{PQPBr}$ and $\mathrm{PQPBr}-2 \mathrm{OH}$ are shown in Figure 1. They showed the combined type I and type IV isotherms. The steep steps of absorption curves at low relative pressure $\left(P / P_{0}<0.01\right)$ indicated the abundance of micropores. The sharp rising adsorptions at high relative pressure regions $\left(0.7<P / P_{0}<1.0\right)$ suggested the existence of macropores, while the presence of the hysteresis loops at $P / P_{0}=0.8-1.0$ regions reflected a certain amount of mesopores. Pore size distribution curve of the representative $\mathrm{PQPBr}-2 \mathrm{OH}$ in Figure 1 (inset) further demonstrated its hierarchically porous structure that was comprised of a large amount of micropores and mesopores, and a small quantity of macropores. For heterogeneous catalysts, the big surface area and high pore volume of the material could maximize the accessibility of the catalytically active sites toward the substrates, while the hierarchically porous structure of the material could enhance the mass transfer of the reactants and products. Therefore, it is not unreasonable to expect that the prepared $\mathrm{PQPBr}-2 \mathrm{OH}$ could be a promising choice of heterogeneous catalyst due to its big surface area, large pore volume, and hierarchical pore structure.

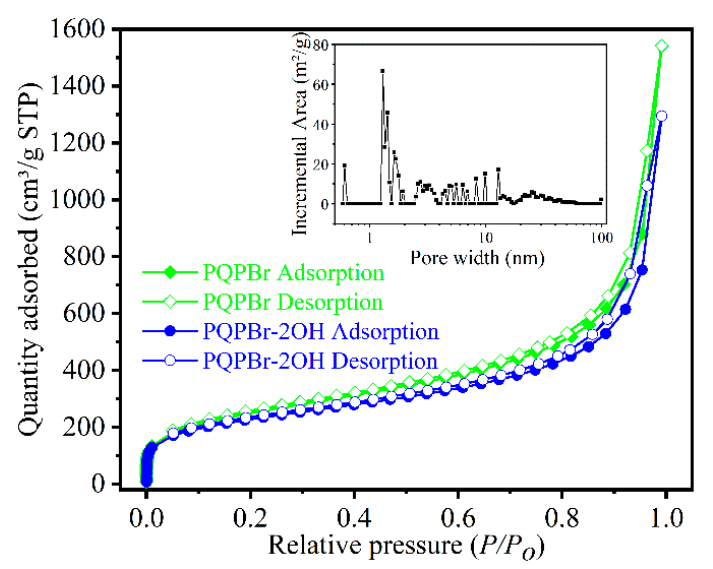

Figure 1. Nitrogen sorption isotherms of $\mathrm{PQPBr}$ and $\mathrm{PQPBr}-2 \mathrm{OH}$, and pore size distribution curve (inset) of $\mathrm{PQPBr}-2 \mathrm{OH}$. 
SEM and TEM were used to study the surface morphologies of PQPBr and PQPBr-2OH. As shown in Figure 2, the representative SEM images of PQPBr and PQPBr-2OH illustrated that the two samples had a similar sponge-like morphology that was composed of small and loosely aggregated particles. TEM images in Figure 2c,d further confirmed the porous and loosely packed morphologies of $\mathrm{PQPBr}$ and PQPBr-2OH.

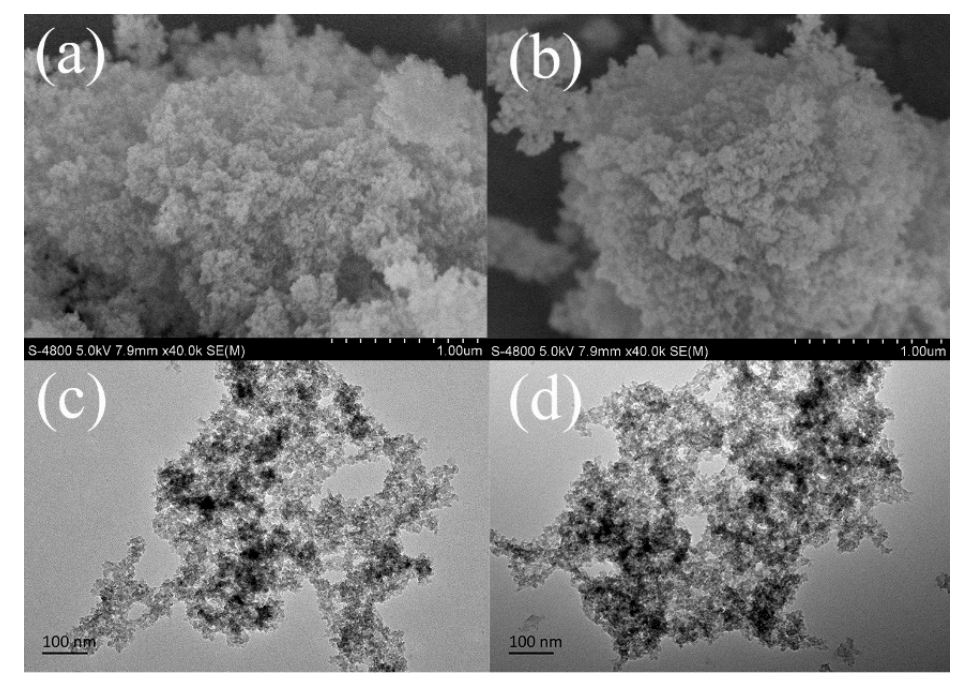

Figure 2. Scanning electron microscope (SEM) images of (a) PQPBr and (b) PQPBr-2OH; Transmission electron microscopy (TEM) image of (c) PQPBr and (d) PQPBr-2OH.

Figure 3 shows the FT-IR spectra of PQPBr, PQPBr-OH, and PQPBr-2OH. The three samples displayed a series of bands at around 2950-2820 and 1600-1450 $\mathrm{cm}^{-1}$, which corresponded to C-H stretching vibrations of the saturated alkyl chains and skeleton stretching of benzene rings, respectively. The FT-IR spectrum of PQPBr in Figure 3a shows a characteristic peak at $1270 \mathrm{~cm}^{-1}$, which could be assigned to the $\mathrm{C}-\mathrm{O}$ stretching vibration of the epoxy groups [49]. After the ring opening reaction in hot water, the characteristic peak at $1270 \mathrm{~cm}^{-1}$ disappeared, and a new characteristic peak of $\mathrm{OH}$ groups at around $1045 \mathrm{~cm}^{-1}$ [50] was present in the FT-IR spectrum of PQPBr-2OH.

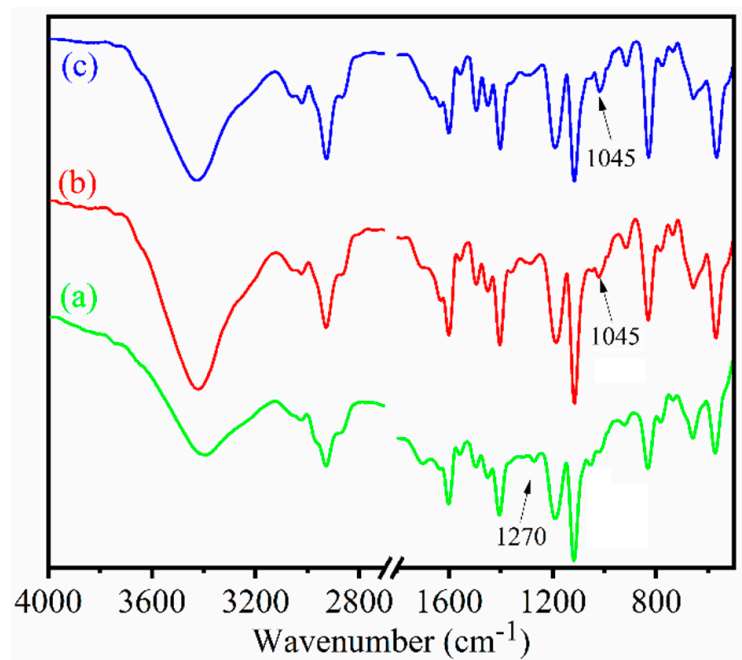

Figure 3. Fourier transformed infrared (FT-IR) spectra of (a) PQPBr, (b) $\mathrm{PQPBr}-\mathrm{OH}$, and (c) $\mathrm{PQPBr}-2 \mathrm{OH}$.

Elemental analysis was used to investigate the elemental contents of the samples. As shown in Table 2, PQPBr showed P and Br contents of $2.06 \mathrm{mmol} / \mathrm{g}$ and $1.78 \mathrm{mmol} / \mathrm{g}$, respectively. As calculated from the mole ratio of $\mathrm{Br} / \mathrm{P}$, about $87 \%$ of phosphine was alkylated with 1-bromo-2,3-epoxypropane in PQPBr. PQPBr showed an $\mathrm{O}$ content of $2.2 \mathrm{mmol} / \mathrm{g}$. After the ring opening reaction in hot water, 
the resulting $\mathrm{PQPBr}-2 \mathrm{OH}$ showed an $\mathrm{O}$ content of $4.3 \mathrm{mmol} / \mathrm{g}$, which was about two times of that in $\mathrm{PQPBr}$. This result indicated that the epoxy groups were almost totally hydrolyzed in PQPBr- $2 \mathrm{OH}$. Elemental analysis of $\mathrm{PQPBr}-\mathrm{OH}$ also suggested that the majority of phosphine was alkylated with 1-bromo-2,3-epoxypropane. Elemental analyses of the three samples showed that the experimental values of $\mathrm{O}$ were slightly higher than the corresponding theoretical values; this deviation might be mainly attributed to the presence of trapped guest molecules (e.g., water), which is common in porous materials.

Table 2. The elemental contents of the prepared samples. ${ }^{\text {a }}$

\begin{tabular}{cccc}
\hline Sample & $\mathbf{P}(\mathbf{m m o l} / \mathbf{g})$ & $\mathbf{B r}(\mathbf{m m o l} / \mathbf{g})$ & $\mathbf{O}(\mathbf{m m o l} / \mathbf{g})$ \\
\hline PQPBr & 2.06 & 1.78 & 2.2 \\
PQPBr-OH & 2.10 & 1.80 & 2.3 \\
PQPBr-2OH & 2.03 & 1.77 & 4.3 \\
\hline \multicolumn{4}{c}{ a Average experimental value of three parallel experiments. }
\end{tabular}

To gain insight into the structural information of the prepared samples, solid-state ${ }^{13} \mathrm{C} N \mathrm{NR}$, solid-state ${ }^{31} \mathrm{P}$ NMR, and XPS analyses were carried out. The solid-state ${ }^{13} \mathrm{C}$ NMR spectra of PQPBr and PQPBr-2OH in Figure 4a displayed a number of peaks at around 120-150 and 30-70 ppm, corresponding respectively to the carbons of benzene rings and saturated carbon chains. Solid-state ${ }^{31} \mathrm{P}$ NMR spectra of $\mathrm{PQPBr}$ and $\mathrm{PQPBr}-2 \mathrm{OH}$ in Figure $4 \mathrm{~b}$ showed a main peak with a small peak at around $19.8 \mathrm{ppm}$ and $-7.7 \mathrm{ppm}$, respectively. The peak at $19.8 \mathrm{ppm}$ can be attributed to the phosphorus atoms of the phosphonium salts [45,51], while the small peak at around $-7.7 \mathrm{ppm}$ should be assigned to a small amount of unfunctionalized phosphine [44,51]. These findings further indicated that most of $\mathrm{P}$ atoms in $\mathrm{PQPBr}$ and PQPBr-2OH was successfully alkylated by alkyl bromide. The XPS full spectra of PQPBr, $\mathrm{PQPBr}-\mathrm{OH}$, and $\mathrm{PQPBr}-2 \mathrm{OH}$ in Figure 4c confirmed the presence of $\mathrm{O}, \mathrm{C}, \mathrm{Br}$, and P elements in the three samples. Compared to $\mathrm{PQPBr}, \mathrm{PQPBr}-2 \mathrm{OH}$ exhibited a higher relative intensity of the $\mathrm{O} 1 \mathrm{~s}$ band, thus verifying its relatively higher O content. P2p XPS spectra of $\mathrm{PQPBr}$ and $\mathrm{PQPBr}-2 \mathrm{OH}$ in Figure $4 \mathrm{~d}$ displayed a characteristic band of phosphonium salt at $132.2 \mathrm{eV}$. The small band $(130.0 \mathrm{eV})$ near the main energy band gap might be assigned to a small amount of unfunctionalized $\mathrm{PPh}_{3}$ units. Thus, solid-state ${ }^{31} \mathrm{P}$ NMR and XPS analyses further demonstrated the successful fabrication of dual hydroxyls-functionalized porous ionic polymers (PQPBr-2OH). Scanning electron microscopy (SEM) elemental mapping was used to investigate the elemental dispersion of the $\mathrm{PQPBr}-2 \mathrm{OH}$ sample. As shown in Figure 5, P, Br, and $\mathrm{O}$ elements were well distributed in the $\mathrm{PQPBr}-2 \mathrm{OH}$ polymer with high degrees of dispersion.

Thermal stability of the sample was examined by thermogravimetric analysis (TGA) over a temperature range of 30 to $600{ }^{\circ} \mathrm{C}$ at a heating rate of $10^{\circ} \mathrm{C} / \mathrm{min}$ under $\mathrm{Ar}$ atmosphere. As depicted in Figure 6, $\mathrm{PQPBr}-\mathrm{OH}$ and $\mathrm{PQPBr}-2 \mathrm{OH}$ showed similar TGA curves. The initial weight loss that occurred at $25-150{ }^{\circ} \mathrm{C}$ should be mainly ascribed to the removal of guest molecules. The rapid weight losses took place at above $200{ }^{\circ} \mathrm{C}$, suggesting that the two samples could be stable at least up to a temperature no less than $200^{\circ} \mathrm{C}$. 

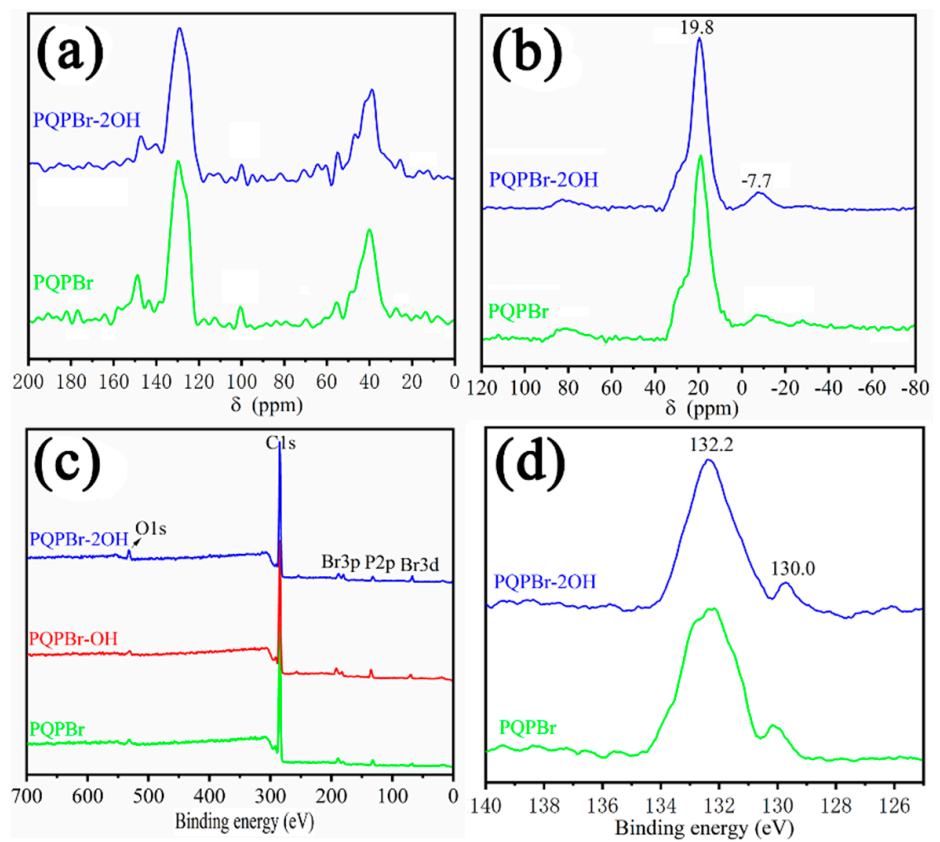

Figure 4. (a) Solid-state ${ }^{13} \mathrm{C}$ nuclear magnetic resonance (NMR) spectra of PQPBr and PQPBr-2OH; (b) solid-state ${ }^{31} \mathrm{P}$ NMR spectra of PQPBr and PQPBr-2OH; (c) XPS full spectra of PQPBr, PQPBr-OH and PQPBr-2OH; (d) P 2p XPS spectra of PQPBr and PQPBr-2OH.

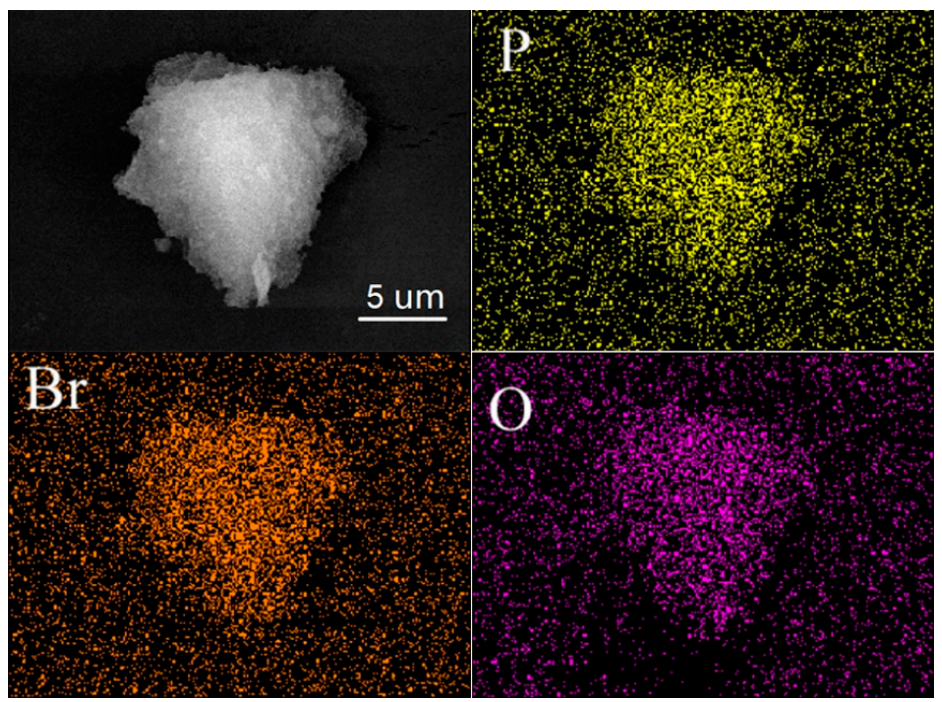

Figure 5. SEM-mapping of PQPBr-2OH. 


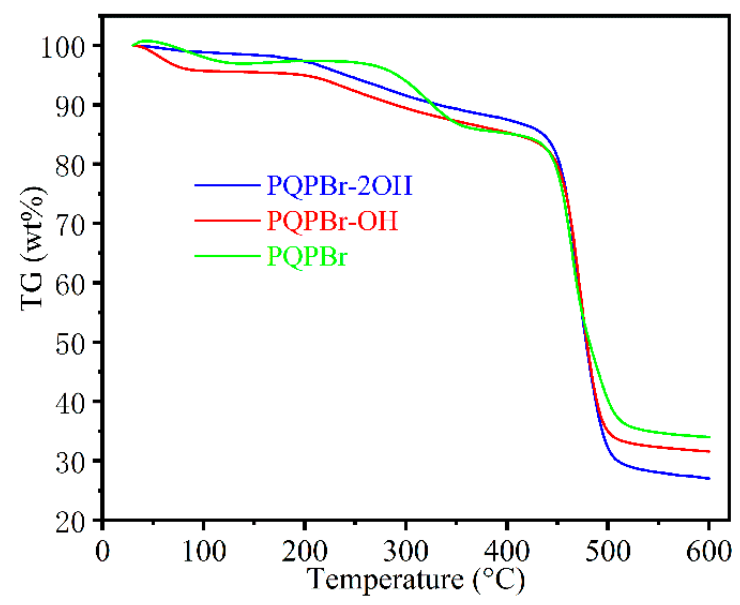

Figure 6. Thermogravimetric analysis (TGA) curves of $\mathrm{PQPBr}, \mathrm{PQPBr}-\mathrm{OH}$, and $\mathrm{PQPBr}-2 \mathrm{OH}$.

\subsection{Catalytic Activity}

With the expected materials in hand, their catalytic performances for the cycloaddition of epoxides with $\mathrm{CO}_{2}$ were investigated. Firstly, the catalytic activities of different catalysts were first investigated using the cycloaddition of inactive styrene oxide as a model reaction. As shown in Table 3, under the mild reaction conditions of $0.5 \mathrm{MPa} \mathrm{CO}$ pressure, $100{ }^{\circ} \mathrm{C}$, and $0.5 \mathrm{~mol} \%$ catalyst loading, $\mathrm{PQPBr}$, $\mathrm{PQPBr}-\mathrm{OH}$, and $\mathrm{PQPBr}-2 \mathrm{OH}$ could promote the cycloaddition of inactive styrene oxide in $41 \%, 75 \%$, and $89 \%$ yields, respectively (Table 3, entries $1-3$ ). It was obvious that $\mathrm{PQPBr}-2 \mathrm{OH}$ showed higher activity than that of $\mathrm{PQPBr}$ and $\mathrm{PQPBr}-\mathrm{OH}$ catalysts. In terms of their similar surface areas and ionic concentrations, the differences in catalytic activities should be attributed to the promoting effect of HBD groups (hydroxyl groups). PQPBr-2OH, having both $\mathrm{Br}$ anions and hydroxyl groups, could cooperatively activate the epoxide, thus improving catalytic performances significantly [20,26,27,33-38]. Moreover, the concentrations of the hydroxyl groups also affected the catalytic performance, and the $\mathrm{PQPBr}-2 \mathrm{OH}$ catalyst with dual hydroxyls exhibited higher catalytic activity than $\mathrm{PQPBr}-\mathrm{OH}$ with one hydroxyl, suggesting that the $\mathrm{OH}$ groups activated the epoxide more easily through multiple hydrogen bonding and, thus, enhanced catalytic efficiencies [8,52]. Under the same conditions, the nonionic polymer $\mathrm{POP}-\mathrm{PPh}_{3}$ gave a negligible yield of cyclic carbonate, verifying that catalytic active sites originated from the ionic moiety (Table 3, entry 4). Previous reports indicated that the reaction temperature had an important effect on the catalytic performance of the catalysts for the cycloaddition of epoxides with $\mathrm{CO}_{2}$ [33-38]. Therefore, the influence of reaction temperature was studied (Table 3, entries 5-8). With the reaction temperature rising from 60 to $120^{\circ} \mathrm{C}$, the yields of target cyclic carbonate were significantly increased, and an almost quantitative yield was obtained at $120^{\circ} \mathrm{C}$. Further increasing the reaction temperature to $140{ }^{\circ} \mathrm{C}$, a slight decrease in selectivity was observed with a minute amount of 1,2-propanediol generated as a by-product. At $120^{\circ} \mathrm{C}$, a lower catalyst loading was also tested. Interestingly, a $98 \%$ yield of cyclic carbonate and a TOF number of $123 \mathrm{~h}^{-1}$ could be obtained using a low catalyst loading of $0.2 \mathrm{~mol} \%$ (Table 3 , entry 9). 
Table 3. Effects of catalysts and reaction temperature. ${ }^{\mathrm{a}}$

\begin{tabular}{|c|c|c|c|c|c|}
\hline Entry & Sample & $\mathrm{T}\left({ }^{\circ} \mathrm{C}\right)$ & Yield (mol \%) & Selectivity (mol \%) & TOF $\left(h^{-1}\right)$ \\
\hline 1 & PQPBr & 100 & 41 & $>99$ & 20.5 \\
\hline 2 & PQPBr-OH & 100 & 75 & $>99$ & 37.5 \\
\hline 3 & PQPBr-2OH & 100 & 89 & $>99$ & 44.5 \\
\hline 4 & $\widetilde{\mathrm{POP}}-\mathrm{PPh}_{3}$ & 100 & $<3$ & - & - \\
\hline 5 & PQPBr-2OH & 60 & 7 & $>99$ & 3.5 \\
\hline 6 & PQPBr-2OH & 80 & 36 & $>99$ & 18 \\
\hline 7 & PQPBr-2OH & 120 & 99 & $>99$ & 49.5 \\
\hline 8 & PQPBr-2OH & 140 & 98 & 98 & 49 \\
\hline $9^{b}$ & PQPBr-2OH & 120 & 98 & $>99$ & 123 \\
\hline
\end{tabular}

${ }^{a}$ Reaction conditions: styrene oxide $(1.2 \mathrm{~g}, 10 \mathrm{mmol})$, catalyst $(0.5 \mathrm{~mol} \%), \mathrm{CO}_{2}(0.5 \mathrm{MPa}), 4 \mathrm{~h}, 800 \mathrm{rpm} .{ }^{\mathrm{b}} \mathrm{PQPBr}-2 \mathrm{OH}$ $(0.2 \mathrm{~mol} \%)$.

Next, the scope of the PQPBr-2OH catalyst for the cycloaddition of epoxides and $\mathrm{CO}_{2}$ was investigated with several typical epoxides. As shown in Table 4, with a $0.05 \mathrm{~mol} \%$ catalyst loading, $\mathrm{PQPBr}-2 \mathrm{OH}$ could effectively convert these typical epoxides into the corresponding cyclic carbonates in moderate to high yields. Moreover, despite the relatively high steric hindrance and low-reactivity, cyclohexene oxide could also be smoothly converted at low catalyst loading (Table 4, entries 8 and 9).

Table 4. Cycloaddition of $\mathrm{CO}_{2}$ with various epoxides using $\mathrm{PQPBr}-2 \mathrm{OH}$ as a catalyst. ${ }^{\mathrm{a}}$.

\begin{tabular}{|c|c|c|c|c|c|}
\hline Entry & Substrate & Product & Catalyst Loading (mol \%) & Yield (mol \%) & Selectivity (mol \%) \\
\hline 1 & & & 0.2 & 99 & $>99$ \\
\hline 2 & & & 0.1 & 99 & $>99$ \\
\hline 3 & & & 0.05 & 98 & $>99$ \\
\hline 4 & & & 0.1 & 99 & $>99$ \\
\hline 5 & & & 0.05 & 91 & $>99$ \\
\hline 6 & & & 0.1 & 99 & $>99$ \\
\hline 7 & & & 0.05 & 95 & $>99$ \\
\hline 8 & & & 0.1 & 99 & $>99$ \\
\hline 9 & & & 0.05 & 74 & $>99$ \\
\hline
\end{tabular}

a Reaction conditions: epoxide (10 mmol), $\mathrm{PQPBr}-2 \mathrm{OH}, \mathrm{CO}_{2}(0.5 \mathrm{MPa}), 120^{\circ} \mathrm{C}, 4 \mathrm{~h}, 800 \mathrm{rpm}$.

Then, stability and recyclability of $\mathrm{PQPBr}-2 \mathrm{OH}$ were tested in a ten-run recycling experiment. For facilitating the recycling of $\mathrm{PQPBr}-2 \mathrm{OH}$, catalytic reactions were performed in scaled-up quantities using styrene oxide $(12 \mathrm{~g}, 100 \mathrm{mmol})$ and $\mathrm{PQPBr}-2 \mathrm{OH}(0.11 \mathrm{~g}, 0.2 \mathrm{~mol} \%)$. Noticeably, even with ten times the amount of styrene oxide, a $99 \%$ yield of target cyclic carbonate could be gained under the reaction conditions. After the first reaction, the solid catalyst was recovered by centrifugation, washed with ethanol, dried under vacuum, and then directly reused in a new catalytic run. The results in Figure 7a revealed that $\mathrm{PQPBr}-2 \mathrm{OH}$ exhibited excellent reusability for the cycloaddition of styrene oxide and $\mathrm{CO}_{2}$. After reusing it 10 times, a 95\% yield of target cyclic carbonate was still preserved. Moreover, the chemical structure and morphology of the recycling $\mathrm{PQPBr}-2 \mathrm{OH}$ after reusing ten times were analyzed by FT-IR, SEM, and $\mathrm{N}_{2}$ adsorption-desorption (Figure $7 \mathrm{~b}-\mathrm{d}$ ). The results suggested 
that the chemical structure and morphology of the recycling $\mathrm{PQPBr}-2 \mathrm{OH}$ were well maintained, thus demonstrating the robustness of the prepared catalyst.
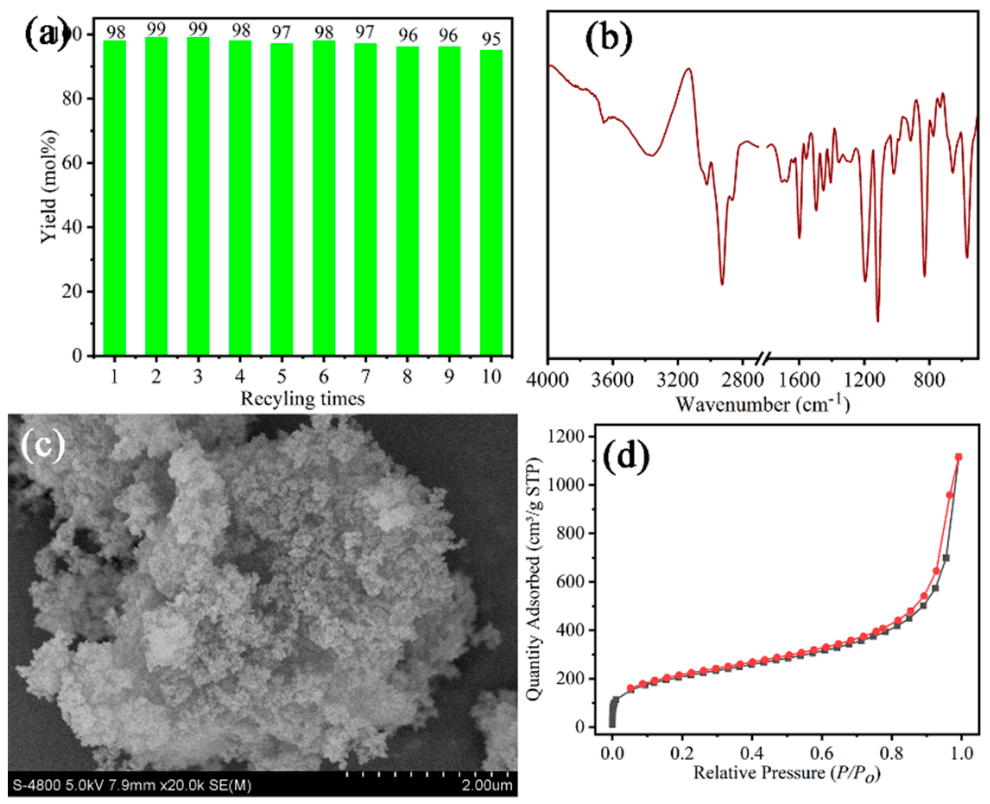

Figure 7. (a) Catalytic reusability of PQPBr-2OH. Characterizations of the recycling of the PQPBr- $2 \mathrm{OH}$ catalyst after reusing it ten times: (b) FT-IR, (c) SEM, and (d) Nitrogen sorption isotherms.

\subsection{Plausible Reaction Mechanism}

According to the above results, $\mathrm{PQPBr}-2 \mathrm{OH}$ with hydroxyl groups exhibited higher catalytic activity than that with non-hydroxyl groups. These findings indicated that hydroxyl groups have an important promoting effect for the cycloaddition of $\mathrm{CO}_{2}$ to epoxide. Moreover, the nucleophilic attack of bromide anion on epoxide is essential. Previous reports suggested that the ring-opening step of epoxide is rate-determining [34,53,54], and a nucleophilic attack of an anion on the carbon atom of epoxide with a synergetic electrophilic attack of a cation on the oxygen atom of epoxide can facilitate this step significantly [20,26,27,33-38]. Moreover, it was also well demonstrated that the hydrogen bond between the hydroxyl group and epoxide has a good promoting effect for the ring-opening step, thus accelerating this cycloaddition reaction [34-38,40,46,52,53]. On the basis of the experimental results and these pioneering reports, a plausible mechanism was proposed and shown in Figure 8. First, hydrogen bonding was formed between the hydroxyl (any one of the dual hydroxyls) of $\mathrm{QPBr}-2 \mathrm{OH}$ and the oxygen atom of epoxide. Then, the bromide anion of $\mathrm{PQPBr}-2 \mathrm{OH}$ attacked the less sterically hindered carbon atom of the epoxide, thus opening the epoxy ring to form a haloalkoxy intermediate. Thereafter, haloalkoxy intermediate reacted with $\mathrm{CO}_{2}$ to produce an alkyl carbonate, which subsequently transformed to cyclic carbonate via a ring-closure step. 


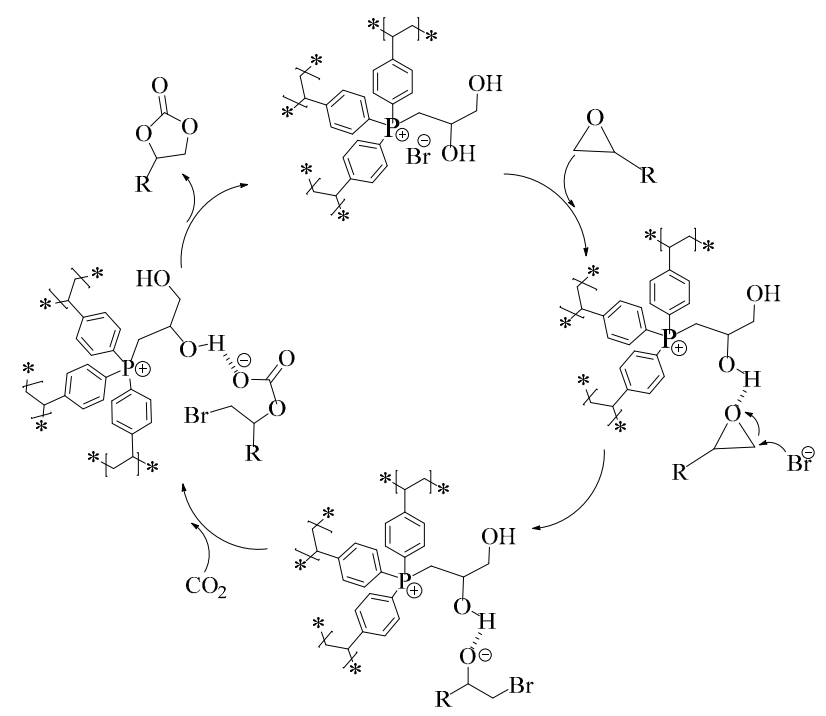

Figure 8. Proposed reaction mechanism for the cycloaddition of $\mathrm{CO}_{2}$ to epoxides catalyzed by $\mathrm{PQPBr}-2 \mathrm{OH}$.

\section{Conclusions}

In summary, a phosphonium-based porous ionic polymer (PQPBr-2OH) with a large surface area, hierarchical pore structure, functional hydroxyl groups, and high density of active sites was developed as an efficient and robust catalyst for the cycloaddition of epoxides with $\mathrm{CO}_{2}$. Control experiments based on the reference catalysts revealed the enhancement achieved by the introduction of dual hydroxyl functionalities in PQPBr-2OH catalyst. Thus, with a low catalyst loading, high yields of cyclic carbonates were obtained under a low $\mathrm{CO}_{2}$ pressure. In addition, $\mathrm{PQPBr}-2 \mathrm{OH}$ was quite robust and recyclable and could be reused at least ten times without obvious change of chemical structure and morphology. Thus, this study provided not only a promising catalyst for the cycloaddition of epoxides with $\mathrm{CO}_{2}$, but also some clues to develop bifunctional and robust phosphonium-based PIPs for $\mathrm{CO}_{2}$ fixation.

Author Contributions: Y.L. and Y.W. conceived and designed the experiments, prepared the draft manuscript; W.Z. and Z.Y. performed the experiments and analysis tools; D.L. revised the manuscript. All authors have read and agreed to the published version of the manuscript.

Funding: This research was funded by the National Natural Science Foundation of China (21763017), the Science and Technology Fund Project of Guizhou Province (qiankehejichu [2018]1414 and qiankehepingtairencai [2019]5604 hao), the Scientific and Technological Innovation Platform of Liupanshui (52020-2018-03-02 and 52020-2017-02-02).

Conflicts of Interest: The authors declare no conflict of interest.

\section{References}

1. Liang, J.; Huang, Y.B.; Cao, R. Metal-organic frameworks and porous organic polymers for sustainable fixation of carbon dioxide into cyclic carbonates. Coord. Chem. Rev. 2019, 378, 32-65. [CrossRef]

2. Ma, Y.; Wang, Z.; Xu, X.; Wang, J. Review on porous nanomaterials for adsorption and photocatalytic conversion of $\mathrm{CO}_{2}$. Chin. J. Catal. 2017, 38, 1956-1969. [CrossRef]

3. $\mathrm{Li}, \mathrm{X} . ; \mathrm{Yu}, \mathrm{J} . ;$ Jaroniec, M.; Chen, X. Cocatalysts for selective photoreduction of $\mathrm{CO}_{2}$ into solar fuels. Chem. Rev. 2019, 119, 3962-4179. [CrossRef] [PubMed]

4. Artz, J.; Müller, T.E.; Thenert, K.; Kleinekorte, J.; Meys, R.; Sternberg, A.; Bardow, A.; Leitner, W. Sustainable conversion of carbon dioxide: An integrated review of catalysis and life cycle assessment. Chem. Rev. 2018, 118, 434-504. [CrossRef] [PubMed]

5. Shaikh, R.R.; Pornpraprom, S.; D'Elia, V. Catalytic strategies for the cycloaddition of pure, diluted, and waste $\mathrm{CO}_{2}$ to epoxides under ambient conditions. ACS Catal. 2018, 8, 419-450. [CrossRef] 
6. Xu, W.; Chen, H.; Jie, K.; Yang, Z.; Li, T.; Dai, S. Entropy-driven mechanochemical synthesis of polymetallic zeolitic imidazolate frameworks for $\mathrm{CO}_{2}$ fixation. Angew. Chem. Int. Ed. 2019, 58, 5018-5022. [CrossRef]

7. Yan, X.M.; Ding, X.; Pan, Y.; Xu, X.W.; Hao, C.; Zheng, W.J.; He, G.H. Quaternary-ammonium-immobilized polystyrenes as efficient and reusable heterogeneous catalysts for synthesis of cyclic carbonate: Effects of linking chains and pendent hydroxyl group. Chin. J. Catal. 2017, 38, 862-871. [CrossRef]

8. Peng, J.; Wang, S.; Yang, H.J.; Ban, B.; Wei, Z.; Wang, L.; Lei, B. Highly efficient fixation of carbon dioxide to cyclic carbonates with new multi-hydroxyl bis-(quaternary ammonium) ionic liquids as metal-free catalysts under mild conditions. Fuel 2018, 224, 481-488. [CrossRef]

9. Ziaee, M.A.; Tang, Y.; Zhong, H.; Tian, D.; Wang, R. Urea-functionalized imidazolium-based ionic polymer for chemical conversion of $\mathrm{CO}_{2}$ into organic carbonates. ACS Sustain. Chem. Eng. 2019, 7, 2380-2387. [CrossRef]

10. Buaki-Sogó, M.; Vivian, A.; Bivona, L.A.; García, H.; Gruttadauria, M.; Aprile, C. Imidazolium functionalized carbon nanotubes for the synthesis of cyclic carbonates: Reducing the gap between homogeneous and heterogeneous catalysis. Catal. Sci. Technol. 2016, 6, 8418-8427. [CrossRef]

11. Xie, Y.; Liang, J.; Fu, Y.; Lin, J.; Wang, H.; Tu, S.; Li, J. Poly (ionic liquid) s with high density of nucleophile/electrophile for $\mathrm{CO}_{2}$ fixation to cyclic carbonates at mild conditions. J. $\mathrm{CO}_{2}$ Utiliz. 2019, 32, 281-289. [CrossRef]

12. Toda, Y.; Komiyama, Y.; Esaki, H.; Fukushima, K.; Suga, H. Methoxy groups increase reactivity of bifunctional tetraarylphosphonium salt catalysts for carbon dioxide fixation: A mechanistic study. J. Org. Chem. 2019, 84, 15578-15589. [CrossRef] [PubMed]

13. Macarie, L.; Simulescu, V.; Ilia, G. Phosphonium-Based Ionic Liquids Used as Reagents or Catalysts. ChemistrySelect 2019, 4, 9285-9299. [CrossRef]

14. Steinbauer, J.; Longwitz, L.; Frank, M.; Epping, J.; Kragl, U.; Werner, T. Immobilized bifunctional phosphonium salts as recyclable organocatalysts in the cycloaddition of $\mathrm{CO}_{2}$ and epoxides. Green Chem. 2017, 19, 4435-4445. [CrossRef]

15. Tahir, N.; Krishnaraj, C.; Leus, K.; Van Der Voort, P. Development of covalent triazine frameworks as heterogeneous catalytic supports. Polymers 2019, 11, 1326. [CrossRef]

16. Kramer, S.; Bennedsen, N.R.; Kegnæs, S. Porous organic polymers containing active metal centers as catalysts for synthetic organic chemistry. ACS Catal. 2018, 8, 6961-6982. [CrossRef]

17. Bhanja, P.; Modak, A.; Bhaumik, A. Porous organic polymers for $\mathrm{CO}_{2}$ storage and conversion reactions. ChemCatChem 2019, 11, 244-257. [CrossRef]

18. Liu, Z.W.; Han, B.H. Ionic porous organic polymers for $\mathrm{CO}_{2}$ capture and conversion. Curr. Opin. Green Sustain. Chem. 2019, 16, 20-25. [CrossRef]

19. Zhang, Y.; Liu, K.; Wu, L.; Zhong, H.; Luo, N.; Zhu, Y.; Tong, M.; Long, Z.; Chen, G. Silanol-enriched viologen-based ionic porous hybrid polymers for efficient catalytic $\mathrm{CO}_{2}$ fixation into cyclic carbonates under mild conditions. ACS Sustain. Chem. Eng. 2019, 7, 16907-16916. [CrossRef]

20. Liu, M.; Wang, X.; Jiang, Y.; Sun, J.; Arai, M. Hydrogen bond activation strategy for cyclic carbonates synthesis from epoxides and $\mathrm{CO}_{2}$ : Current state-of-the art of catalyst development and reaction analysis. Catal. Rev. 2019, 61, 214-269. [CrossRef]

21. Dai, Z.; Sun, Q.; Chen, F.; Pan, S.; Wang, L.; Meng, X.; Li, J.; Xiao, F.S. Enhancement of catalytic activity in epoxide hydration by increasing the concentration of cobalt (III)/salen in porous polymer catalysts. ChemCatChem 2016, 8, 812-817. [CrossRef]

22. Chen, Y.; Luo, R.; Xu, Q.; Jiang, J.; Zhou, X.; Ji, H. Charged metalloporphyrin polymers for cooperative synthesis of cyclic carbonates from $\mathrm{CO}_{2}$ under ambient conditions. ChemSusChem 2017, 10, 2534-2541. [CrossRef] [PubMed]

23. Chen, J.; Zhong, M.; Tao, L.; Liu, L.; Jayakumar, S.; Li, C.; Li, H.; Yang, Q. The cooperation of porphyrin-based porous polymer and thermal-responsive ionic liquid for efficient $\mathrm{CO}_{2}$ cycloaddition reaction. Green Chem. 2018, 20, 903-911. [CrossRef]

24. Li, J.; Han, Y.; Lin, H.; Wu, N.; Li, Q.; Jiang, J.; Zhu, J. Cobalt-salen based porous ionic polymer: The role of valence for cooperative conversion of $\mathrm{CO}_{2}$ to cyclic carbonate. ACS Appl. Mater. Interfaces 2020, 12, 609-618. [CrossRef]

25. Li, J.; Han, Y.; Ji, T.; Wu, N.; Lin, H.; Jiang, J.; Zhu, J. Porous metallosalen hypercrosslinked ionic polymers for cooperative $\mathrm{CO}_{2}$ cycloaddition conversion. Ind. Eng. Chem. Res. 2020, 59, 676-684. [CrossRef] 
26. Xu, D.; Guo, J.; Yan, F. Porous ionic polymers: Design, synthesis, and applications. Prog. Polym. Sci. 2018, 79, 121-143. [CrossRef]

27. Sun, J.-K.; Antonietti, M.; Yuan, J. Nanoporous ionic organic networks: From synthesis to materials applications. Chem. Soc. Rev. 2016, 45, 6627-6656. [CrossRef]

28. Chen, Y.; Luo, R.; Bao, J.; Xu, Q.; Jiang, J.; Zhou, X.; Ji, H. Function-oriented ionic polymers having high-density active sites for sustainable carbon dioxide conversion. J. Mater. Chem. 2018, 6, 9172-9182. [CrossRef]

29. Zhang, Y.; Chen, G.; Wu, L.; Liu, K.; Zhong, H.; Long, Z.; Tong, M.; Yang, Z.; Dai, S. Two-in-one: Construction of hydroxyl and imidazolium-bifunctionalized ionic networks in one-pot toward synergistic catalytic $\mathrm{CO}_{2}$ fixation. Chem. Commun. 2020. [CrossRef]

30. Li, J.; Jia, D.; Guo, Z.; Liu, Y.; Lyu, Y.; Zhou, Y.; Wang, J. Imidazolinium based porous hypercrosslinked ionic polymers for efficient $\mathrm{CO}_{2}$ capture and fixation with epoxides. Green Chem. 2017, 19, 2675-2686. [CrossRef]

31. Zhang, W.; Ma, F.; Ma, L.; Zhou, Y.; Wang, J. Imidazolium-functionalized ionic hypercrosslinked porous polymers for efficient synthesis of cyclic carbonates from simulated flue gas. ChemSusChem 2020, 13, 341-350. [CrossRef] [PubMed]

32. Wang, W.; Li, C.; Yan, L.; Wang, Y.; Jiang, M.; Ding, Y. Ionic liquid/Zn-PPh 3 integrated porous organic polymers featuring multifunctional sites: Highly active heterogeneous catalyst for cooperative conversion of $\mathrm{CO}_{2}$ to cyclic carbonates. ACS Catal. 2016, 6, 6091-6100. [CrossRef]

33. Chen, G.; Zhang, Y.; Xu, J.; Liu, X.; Liu, K.; Tong, M.; Long, Z. Imidazolium-based ionic porous hybrid polymers with POSS-derived silanols for efficient heterogeneous catalytic $\mathrm{CO}_{2}$ conversion under mild conditions. Chem. Eng. J. 2020, 381, 122765. [CrossRef]

34. Guo, Z.; Jiang, Q.; Shi, Y.; Li, J.; Yang, X.; Hou, W.; Zhou, Y.; Wang, J. Tethering dual hydroxyls into mesoporous poly (ionic liquid) s for chemical fixation of $\mathrm{CO}_{2}$ at ambient conditions: A combined experimental and theoretical study. ACS Catal. 2017, 7, 6770-6780. [CrossRef]

35. Anthofer, M.H.; Wilhelm, M.E.; Cokoja, M.; Drees, M.; Herrmann, W.A.; Kühn, F.E. Hydroxy-functionalized imidazolium bromides as catalysts for the cycloaddition of $\mathrm{CO}_{2}$ and epoxides to cyclic carbonates. ChemCatChem 2015, 7, 94-98. [CrossRef]

36. Wang, X.; Li, J.; Chen, G.; Guo, Z.; Zhou, Y.; Wang, J. Hydrophobic mesoporous poly(ionic liquid)s towards highly efficient and contamination-resistant solid-base catalysts. Chem CatChem 2015, 7, 993-1003. [CrossRef]

37. Bernard, F.L.; Duczinski, R.B.; Rojas, M.F.; Fialho, M.C.C.; Carreno, L.A.; Chaban, V.; Vecchia, F.D.; Einloft, S. Cellulose based poly (ionic liquids): Tuning cation-anion interaction to improve carbon dioxide sorption. Fuel 2018, 211, 76-86. [CrossRef]

38. Dai, W.L.; Chen, L.; Yin, S.F.; Luo, S.L.; Au, C.T. 3-(2-Hydroxyl-ethyl)-1-propylimidazolium bromide immobilized on SBA-15 as efficient catalyst for the synthesis of cyclic carbonates via the coupling of carbon dioxide with epoxides. Catal. Lett. 2010, 135, 295-304. [CrossRef]

39. Dai, W.-L.; Jin, B.; Luo, S.-L.; Luo, X.-B.; Tu, X.-M.; Au, C.-T. Functionalized phosphonium-based ionic liquids as efficient catalysts for the synthesis of cyclic carbonate from epoxides and carbon dioxide. Appl. Catal. Gen. 2014, 470, 183-188.

40. Dai, W.; Zhang, Y.; Tan, Y.; Luo, X.; Tu, X. Reusable and efficient polymer nanoparticles grafted with hydroxyl-functionalized phosphonium-based ionic liquid catalyst for cycloaddition of $\mathrm{CO}_{2}$ with epoxides. Appl. Catal. Gen. 2016, 514, 43-50. [CrossRef]

41. Zhang, Q.; Zhang, S.; Li, S. Novel functional organic network containing quaternary phosphonium and tertiary phosphorus. Macromolecules 2012, 45, 2981-2988. [CrossRef]

42. Wang, J.; Yang, J.G.W.; Yi, G.; Zhang, Y. Phosphonium salt incorporated hypercrosslinked porous polymers for CO 2 capture and conversion. Chem. Commun. 2015, 51, 15708-15711. [CrossRef] [PubMed]

43. Sun, Q.; Jin, Y.; Aguila, B.; Meng, X.; Ma, S.; Xiao, F.S. Porous ionic polymers as a robust and efficient platform for capture and chemical fixation of atmospheric $\mathrm{CO}_{2}$. ChemSusChem 2017, 10, 1160-1165. [CrossRef] [PubMed]

44. Hu, K.; Tang, Y.; Cui, J.; Gong, Q.; Hu, C.; Wang, S.; Dong, K.; Meng, X.; Sun, Q.; Xiao, F.S. Location matters: Cooperativity of catalytic partners in porous organic polymers for enhanced $\mathrm{CO}_{2}$ transformation. Chem. Commun. 2019, 55, 9180-9183. [CrossRef] [PubMed]

45. Wan, Y.; Song, F.; Ye, T.; Li, G.; Liu, D.; Lei, Y. Carbonylative Suzuki coupling and alkoxycarbonylation of aryl halides using palladium supported on phosphorus-doped porous organic polymer as an active and robust catalyst. Appl. Organometal. Chem. 2019, 33, e4714. [CrossRef] 
46. Chen, G.J.; Zhou, Y.; Zhao, P.P.; Long, Z.Y.; Wang, J. Mesostructured dihydroxy-functionalized guanidinium-based polyoxometalate with enhanced heterogeneous catalytic activity in epoxidation. ChemPlusChem 2013, 78, 561-569. [CrossRef]

47. Sun, Q.; Dai, Z.; Liu, X.; Sheng, N.; Deng, F.; Meng, X.; Xiao, F.S. Highly efficient heterogeneous hydroformylation over Rh-metalated porous organic polymers: Synergistic effect of high ligand concentration and flexible framework. J. Am. Chem. Soc. 2015, 137, 5204-5209. [CrossRef]

48. Sun, Q.; Jiang, M.; Shen, Z.; Jin, Y.; Pan, S.; Wang, L.; Meng, X.; Chen, W.; Ding, Y.; Li, J.; et al. Porous organic ligands (POLs) for synthesizing highly efficient heterogeneous catalysts. Chem. Commun. 2014, 50, 11844-11847. [CrossRef]

49. Wang, D.H.; Sihn, S.; Roy, A.K.; Baek, J.B.; Tan, L.S. Nanocomposites based on vapor-grown carbon nanofibers and an epoxy: Functionalization, preparation and characterization. Eur. Polym. J. 2010, 46, 1404-1416. [CrossRef]

50. Cai, J.; Ma, H.; Zhang, J.; Du, Z.; Huang, Y.; Gao, J.; Xu, J. Catalytic oxidation of glycerol to tartronic acid over $\mathrm{Au} / \mathrm{HY}$ catalyst under mild conditions. Chin. J. Catal. 2014, 35, 1653-1660. [CrossRef]

51. Lei, Y.; Chen, Z.; Lan, G.; Wang, R.; Zhou, X.Y. Pd nanoparticles stabilized by phosphine-functionalized porous ionic polymer for efficient catalytic hydrogenation of nitroarenes in water. New J. Chem. 2020. [CrossRef]

52. Liu, M.; Li, X.; Liang, L.; Sun, J. Protonated triethanolamine as multi-hydrogen bond donors catalyst for efficient cycloaddition of $\mathrm{CO}_{2}$ to epoxides under mild and cocatalyst-free conditions. J. $\mathrm{CO}_{2}$ Util. 2016, 16, 384-390. [CrossRef]

53. Foltran, S.; Mereau, R.; Tassaing, T. Theoretical study on the chemical fixation of carbon dioxide with propylene oxide catalyzed by ammonium and guanidinium salts. Catal. Sci. Technol. 2014, 4, 1585-1597. [CrossRef]

54. Sun, H.; Zhang, D.J.J. Density functional theory study on the cycloaddition of carbon dioxide with propylene oxide catalyzed by alkylmethylimidazolium chlorine ionic liquids. Phys. Chem. 2007, 111, 8036-8043. [CrossRef] [PubMed] 